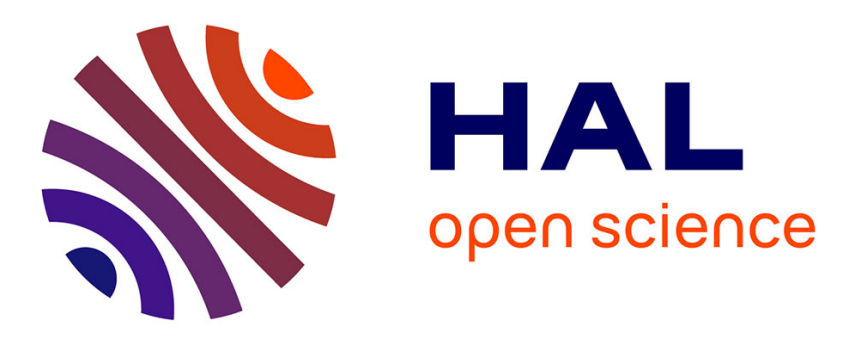

\title{
Towards Opportunistic Service Provisioning in Intermittently Connected Hybrid Networks
}

\author{
Ali Makke, Yves Mahéo, Nicolas Le Sommer
}

\section{To cite this version:}

Ali Makke, Yves Mahéo, Nicolas Le Sommer. Towards Opportunistic Service Provisioning in Intermittently Connected Hybrid Networks. 4th International Conference on Networking and Distributed Computing (ICNDC 2013), Dec 2013, Honk Kong, China. pp.28-32, 10.1109/ICNDC.2013.30 . hal00916297

\section{HAL Id: hal-00916297 \\ https://hal.science/hal-00916297}

Submitted on 7 Nov 2014

HAL is a multi-disciplinary open access archive for the deposit and dissemination of scientific research documents, whether they are published or not. The documents may come from teaching and research institutions in France or abroad, or from public or private research centers.
L'archive ouverte pluridisciplinaire HAL, est destinée au dépôt et à la diffusion de documents scientifiques de niveau recherche, publiés ou non, émanant des établissements d'enseignement et de recherche français ou étrangers, des laboratoires publics ou privés. 


\title{
Towards Opportunistic Service Provisioning in Intermittently Connected Hybrid Networks
}

\author{
Ali Makke, Yves Mahéo and Nicolas Le Sommer \\ IRISA, Université de Bretagne-Sud, France
}

\begin{abstract}
An intermittently connected mobile ad hoc hybrid network (ICHN) is one of the challenging networks induced by the proliferation of handheld devices equipped with wireless interfaces. It is formed by a number of fixed infostations, hosting service providers, and some mobile devices, hosting service clients, organized in an ad hoc network facing frequent connectivity disruptions. This paper focuses on two novel protocols that form the basis of a service provisioning platform specifically designed for ICHN. It details a service discovery protocol and a service invocation protocol that implement in particular an opportunistic routing based on a time-aware heuristic. Performance evaluation shows that the specialization of TAO is able to guarantee good performance with limited overhead.
\end{abstract}

Index Terms-Opportunistic Networks, Disconnected Mobile Ad hoc Networks, Service Oriented Computing

\section{INTRODUCTION}

The amount and the variety of wireless communicating devices affecting our daily life is noticeably increasing. Many of these devices can communicate directly with one another, and to support multi-hop communication between the various devices that may simultaneously appear in the environment, a Mobile Ad Hoc Network (MANET) could be simply created by relying only on the collaboration among these devices and without resorting to any pre-existing infrastructure of communication. However the underlying assumption that a MANET is dense enough to form quasi-permanently a fully connected graph often proves to be unrealistic. In practice, the free movement of people coupled with the short communication range of wireless interfaces result in unpredictable disruptions in the communication links. These disruptions entail the creation of disconnected communication islands, forming a type of network known as Intermittently Connected Mobile Ad Hoc Network (ICMANET). In these conditions, it is difficult, and most often impossible, to maintain an endto-end path between two devices thanks to traditional routing protocols designed for wired networks or thanks to dynamic routing protocols such as AODV or OLSR.

Delay-tolerant networking and-more specifically for ICMANETs - opportunistic networking, have emerged as sets of techniques to cope with these issues. Hence, a number of routing protocols have been proposed in the last few years for networks that suffer from frequent and unpredictable disruptions, by implementing the "store, carry and forward" general principle. When two devices cannot communicate directly because they are not simultaneously present in the same network island, these protocols make it possible to exploit other mobile nodes as intermediate relays that can carry a copy of a message when they move and forward it afterwards to other nodes so that it eventually reaches its destination.

In the aim of extending the concept of opportunistic networking, the different resources made available by the various devices (static or mobile) that appear in the environment can be exposed as application services. This opens the way to opportunistic computing: following a Service-Oriented Architecture approach, an application can be built on top of a middleware layer that offers means to discover the services provided in the network and invoke the desired services, regardless of the fact that they are in the radio range or remote.

In our opinion, and although it has been seldom studied per se, one of the most interesting and realistic type of network in which opportunistic computing can be applied should be what we call an Intermittently Connected Hybrid Network (ICHN), that is, an infrastructure-based network with opportunistic extensions. An ICHN includes some fixed infostations (potentially connected together with some fixed infrastructure, typically the Internet) and a set of mobile devices, viewed as an ICMANET that is exploited with opportunistic networking techniques. Furthermore, the volatility and the limited resources of the mobile devices induce that the majority of the provided services are offered by the fixed infostations.

In this paper, we present two protocols that form the basis of the Time-Aware Opportunistic middleware (TAO), devoted to service provisioning in ICHNs. Indeed, TAO implements a protocol for service discovery and a protocol for service invocation, combining several well-known heuristics and mechanisms in a coherent platform. The originality of TAO lies in the fact that it takes all the specificities of ICHNs into account, namely the frequent and unpredictable communication disruptions, the mobility of numerous devices, the stability of the service providers, and the interconnection of infostations providing services.

\section{RELATED WORK}

Several research studies have been conducted in the domain of delay-tolerant-networking for MANETS [1] and opportunistic networking [2], but the vast majority focus on communication between mobile nodes and do not exploit the presence of fixed points in the network. In addition, these works consider general communication: sending a message from a node A to a node $\mathrm{B}$, ignoring potential optimizations for communication that follow the request-response pattern of service invocation.

Indeed, several mobility-based approaches that use short range communication and that exploit mobility to deliver 
data have been investigated. Some of them exploit the nonrandomness mobility behavior of special nodes [3] to deliver messages, while others rely on flooding-based approaches [4] to disseminate the messages over the network. On the other hand, several strategies and optimizations to control the replication of messages have been devised, for example by limiting the number of disseminated copies [5]. Some other works have investigated solutions relying on probabilistic and prediction techniques using context properties and history contacts to choose among a set of nodes the best relay(s) for each message that must be forwarded ([6], [7]). History-based algorithms can achieve high efficiency and delivery ratio, but have the major drawback of assuming that a node is able to register a large amount of contact information in its buffers.

As far as service provisioning is concerned, the research works described in the literature essentially focus on service discovery in dense MANETs, very few of them consider ICMANETs (e.g. [8], [9]) and, to our knowledge, none of the latter include fixed infostations in their studies. Despite the fact that these protocols consider the dynamicity of the targeted networks, they mainly assume the availability of a fully-connected underlying network which can support an endto-end path between any two nodes in the network. Obviously, such assumptions cannot be applied over ICHN, where the volatility of the devices, the short communication range of wireless interfaces, accompanied with the radio interference and the free movement of people, result low density networks with no guarantees on the end-to-end paths or on the reliability of communications. The provision of application services with this kind of opportunistic and asynchronous communications has been addressed so far only by a few research works [2], [10], [11], [12]. Mahéo and Said [12] and Conti and Kumar [2] focus on service provisioning in opportunistic networks composed solely of mobile nodes. In [12], the authors propose content-based service discovery and invocation solutions in order to exploit the redundancy of the services offered by the mobile devices that can move freely. Conti and Kumar [2] target networks relying on social interactions between mobile nodes that act as both clients and providers of services. Due to the volatility and the limited resources of the mobile devices, the number of relevant services that can be offered by these devices are limited in comparison to those that could be offered in hybrid networks. Unlike in [12] and [2], in [11] the services are provided by fixed infostations in limited geographic areas. In contrast with the environments we consider, in [11], the infostations are not connected together and nodes are aware of their own location. Finally, most of the above-presented works consider networks formed only of mobile devices. In contrast, TAO also takes into account the presence of some potential fixed devices in the network.

\section{TAO PROTOCOLS}

In this section, we explain how the specificities of ICHNs guide the design of the architecture of TAO, and describe the key aspects of the protocols. TAO is designed as couple of protocols which aim at supporting the discovery and invocation of software services to fulfill the specific requests of the clients roaming an ICHN network. In general, in the aim of accommodating the free mobility patterns of the nodes, both TAO protocols rely on the "store, carry and forward" principle to send and receive messages. Therefore, messages will be stored in the local caches of intermediate nodes, and the mobility of those nodes is exploited to bring messages closer to their destination.

\section{A. Service Discovery in TAO}

Before being able to invoke a service hosted by an infostation, a client must be able to discover the existence of this service as well as its details. The TAO service discovery protocol (TAO-DIS) serves this purpose by allowing a client to automatically obtain the service descriptors produced and advertised by the providers in the network. Such descriptors consist in lists of properties that covers the functional and non functional aspects of service provision.

In ICHNs, infostations may be subject to a form of administrative grouping or clustering. In this paper, we consider that infostations form clusters. The infostations that are affiliated to one cluster are connected together and aware of the services proposed by each of them. Though, these infostations are unaware of the presence of other infostations in the network or of the services they provide.

The fastest way to inform all the nodes of the offered services is to perform an epidemic dissemination of the service advertisements over the whole network. However, this is a very costly operation for it imposes an excessive load on the network. In this respect, TAO-DIS proposes an optimization of the epidemic dissemination approach by benefiting from the compulsory presence of the periodic beaconing mechanism utilized for neighbor discovery in such networks. Indeed, by piggybacking the IDs of the provided services in the beacon messages and exchanging the actual descriptors of the services only when needed, we restrict the number of exchanged messages while maintaining the same dissemination performance. For that, we represent every service by a unique identifier or a "hashkey". Ensuring unique hashkeys is obtained by applying a hash function on the concatenation of the different properties describing the service and the infostation ID providing the service. Thus, for every service descriptor $s_{i}$, the hashkey generation process is defined by:

$K_{s_{i}}=\mathcal{H}($ Functional + NonFunctional + InfostationID $)$

Furthermore, since every infostation (or cluster of infostations) might provide more than one service, we define a "service guide" (SG) as a catalog that lists the descriptors of all the provided services by a cluster of infostations. The main components of the SG are a set of entries each of which details a provided service, the time tag that represents the creation date of this exact version of the SG, the cluster ID, and the unique SG hashkey defined by:

$$
K_{S G}=\mathcal{H}\left(\sum_{i=1}^{i=N} K_{s_{i}}\right)
$$

Where $\sum$ is the string concatenation operation and $s_{i}$ is a service descriptor entry. 
In order to avoid the excessive exchange of unnecessary messages, we introduce the tuple $<S G$ hashkey, Cluster ID, Time Tag $>$ in the header of the beacon messages. Thus, the comparison between the SG hashkey already registered in the local cache of a node and the value received from a neighbor will result in sending the newest version of the SG only if any difference is detected between the two values. This difference requires a reaction only from the node in possession of the newer version of the SG, where this is decided by comparing the two time tags. Therefore, any change in the SG (addition, withdrawal or modification of a service descriptor) will yield a modification of the SG hashkey. As a result, the modified part of the SG is propagated as soon as it happens.

In summary, in TAO-DIS, we perform a controlled and opportunistic dissemination of service advertisements in the network, where the actual exchange of a SG only occurs when two nodes carrying two different versions of the same SG enter in the transmission range of each other.

\section{B. Service Invocation in TAO}

The second part of TAO is an invocation protocol (TAOINV) that allows a mobile client to send a request to a service provider and to receive a response back from this provider following the "store, carry and forward" principle. The primary objective behind the design of TAO-INV is estimating the ability of intermediate nodes to deliver the messages from a client device to the infostations as they represent the main providers of services in an ICHN.

When a client (or an application on the client's device) discovers the various services present in the network, it chooses the service that best matches its needs and generates an "invocation message" to invoke this service on its provider. In order to avoid the too costly epidemic forwarding of the invocation messages in the network, TAO-INV strives to evaluate the neighbors of a node (i.e., the nodes present in its transmission range) so as to classify them as good or bad carriers, in order to determine the number of copies that should be generated and the relay nodes that should contribute in transmitting these messages toward the infostation. A neighbor is considered a good carrier if it is capable of relaying the messages from the client closer to the infostation. In practice, TAO implements a time stamping-based heuristic that classifies a node as a good carrier if its last date of contact with an infostation is more recent than the others.

Consequently, the node sets a threshold equal to its last contact time with the infostation. The neighbors having a more recent contact time with the infostation are classified as good carriers and will receive copies of the invocation messages. In addition, the value of the threshold is augmented and set equal to the value of the most recent contact time of a neighbor with the infostation, in the aim of reducing the dissemination of the invocation messages.

However, as we target highly disrupted networks, it might be sometimes risky to forward copies exclusively to good carriers, especially if the source node is located remotely from an infostation, with a huge gap separating them. Hence, in TAOINV, each mobile node has a stock of a few number of copies dedicated to bad carriers. This number of copies is limited in order to avoid network overload and resource consumption on mobile devices.

As introduced before, the only piece of information needed by TAO-INV to perform the selection process of good and bad carriers is the last contact time of the neighbors with an infostation. It is piggy-backed in the beacon messages that are periodically exchanged among the nodes in the network. By that, we limit the load imposed by TAO-INV on the network and eliminate the exchange of unnecessary messages.

After the infostation has received the invocation message, it generates a "service response" and destines it for the client that requested the service. In order to send the response back toward this client, the infostation relies on the information included in the invocation message it has just received. In fact, the invocation message records in its header the path it has traversed (i.e., the order of the nodes that have contributed in relaying it) to move from the client toward the infostation. Hence, TAO-INV implements a reverse source routing mechanism to build the path that should be traveled back by the response message from the infostation toward the client. If the source routing fails, because an intermediate node becomes unreachable, the carrier of the service response keeps this message in its local cache until encountering any of the nodes mentioned in the header of the service response message. All of the new carriers will try to resume the source routing process.

Finally, TAO-INV makes it possible to control the propagation of messages in the network using two parameters: a lifetime and a number of hops. When the lifetime is expired or when the number of hops is zero, the message is automatically removed from the local cache and will not be forwarded anymore. Moreover, in order to reduce the dissemination of service requests in the network, we have implemented in TAO-INV a network healing mechanism that exploits the request/response paradigm. When a carrier receives a response for a request it has locally, it removes the request from its local cache and stops forwarding this request. Thus, progressively, the request will no longer be forwarded in the network.

\section{EVALUATION}

In this section, we present the service discovery performance evaluation of TAO, discussing the obtained results in details. The metrics measured for TAO-INV are the satisfaction ratio (the number of service requests for which a client node receives a response over the total number of service requests sent by clients) and the service delivery delay (the delay between the emission of the service request and the actual reception of the corresponding service response). As for TAO-DIS, we measured the average dissemination delay (the delay between the emission of the SG by the infostation and its actual reception by the mobile clients). For both TAO-INV and TAO-DIS, we also evaluated the network load, i.e., the overall number of messages exchanged between nodes during the simulation. Only messages carrying data related to the protocols were counted. 


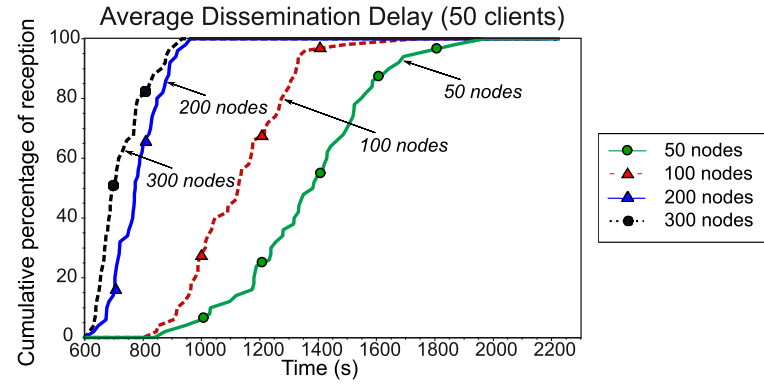

Figure 1. Evaluation of TAO-DIS: Average Dissemination Delay

\section{A. Simulation Setup}

We used the OMNeT++ network simulator [13] to conduct experiments that involved service providers, service clients and simple relay nodes as well as a number of fixed infostations. A first series of simulations exploited purely simulated movements with up to 300 nodes. In an area of $1 \mathrm{~km}^{2}$, a simple hybrid network was deployed, that was composed of one infostation (in the center of the area) and a set of mobile devices carried by pedestrians (moving according to a random way-point mobility model, with a speed set to $0.5 \mathrm{~m} / \mathrm{s}$ ). A subset of these pedestrians acted as clients that requested services from the infostation, the rest of the mobile devices serving as potential relays. The second environment was based on the exploitation of real movement data. We used a data set from the CRAWDAD repository that contains 92 GPS traces of movement of students in a campus [14]. Combined with the use of OMNeT++ simulator, this makes it possible to take into account low-level constraints (MAC level and physical level). plots number of messages over timeFor the sake of comparison with the artificial environment, we added 3 infostations in the $4 \times 4 \mathrm{~km}^{2}$ area, so that the infostations were distant from each other by more than $1 \mathrm{~km}$. Again, roles of clients and relays were assigned to the mobile nodes.

In both simulation environments, the communication range of the mobile devices and of the infostation was set approximately to $30 \mathrm{~m}$. In the experiments aiming at evaluating TAO-INV, the mobile clients invoked the desired services every 3 minutes, omitting some requests randomly. For the evaluation of TAO-DIS, the number of services descriptors produced by the infostation varied between 1 and 3 . We did not assign a lifetime and a maximum number of hops to the messages as these values are directly related with the nature of the provided services and their relative applications. Thus, the number of messages that roam the network increases continuously during the simulation. A warm up period of 10 minutes was used in the beginning of the simulations before the infostation started to generate the SG (respectively before clients started to generate request messages). Each simulation ran then during one hour before the infostation ceased to produce service descriptors (respectively clients ceased to emit invocation requests). Finally, the simulation proceeded for 10 minutes to allow all the messages to be delivered. In these simulations we consider an infinite buffer size for both mobile devices and infostations.
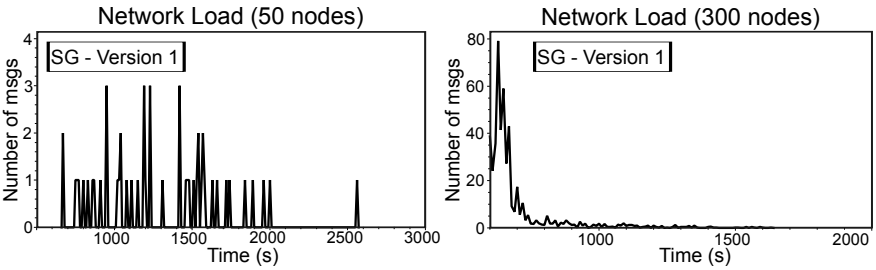

(a) One version of the service guide
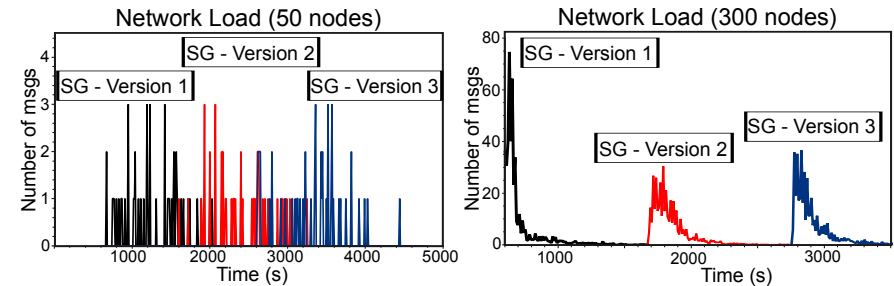

(b) Three versions of the service guide

Figure 2. Evaluation of TAO-DIS: Network Load

\section{B. Evaluation of TAO-DIS}

For a first evaluation of TAO-DIS, we focused on the average delay of dissemination of the SG. The curves in Figure 1 show the cumulative percentage of clients that have received the SG with respect to time, for four densities of mobiles nodes. In this case study, the infostation generated one SG at time $=600$ seconds. The epidemic nature of the dissemination of the SG can be observed (the performance is actually very close to the one of a plain flooding). Expectedly, TAO-DIS can benefit from the impact of the density of the network on the efficiency of the dissemination. For example, to reach $95 \%$ of the nodes, $1150 \mathrm{~s}$ are required with 50 nodes, whereas $275 \mathrm{~s}$ are sufficient with 300 nodes).

In the next experiment we focused our attention on the total volume of traffic generated by all the nodes present in the network in order to ensure a full dissemination of the SG. We considered two case studies: in the first one the infostation generated only one SG at the beginning of the experiment, while in the second one the infostation produced three different service descriptors in three successive steps, thus leading to three different versions of the SG. We introduced a 1000-second time gap between the generation of the service descriptors in order to track the dissemination of each version of the SG. Moreover, in each case study we varied the number of mobile nodes forming the network from 50 to 300 to change the density of the network and thus the rate of contact among the different nodes in the network. Figure 2 plots the evolution of the total number of exchanged SG update messages over time. It is easy to see that, as the number of mobile nodes forming the network increases (from 50 to 300 in our case), the dissemination speed of the SG significantly increases. Nevertheless, we notice that our protocol retains its scalability: the number of exchanged messages remains of the order of the number of nodes roaming the network. On the other hand, coherent with what was observed before, when the density of the network is low, the speed of dissemination of the SG is low as we notice that the exchange of messages was stretched over a larger period of time. These results are 

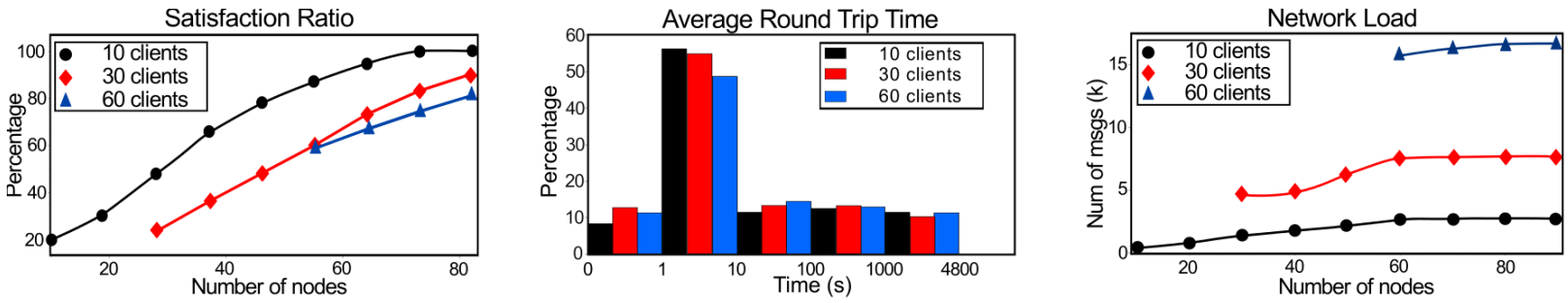

Figure 3. Evaluation of TAO-INV

duplicated in Figure 2b. When having a large number of nodes in the network, the SG was fully disseminated over all the nodes before the creation of the new service descriptor (i.e., the new version of the SG). On the contrary, when having only 50 nodes roaming the network we can clearly see the dissemination of more than one version of the SG at the same period of time. Finally, these results show that the overall load imposed over the network is limited and the algorithm implemented in TAO-DIS ensures a fast dissemination of the SG over all the mobile nodes present in the network.

\section{Evaluation of TAO-INV}

The curves in Figure 3 present the variation of the satisfaction ratio for TAO-INV when the number of mobile nodes in the network increase from 20 to 92 , and the number of clients from 20 to 60 while moving according to the real movement traces. The interesting point to observe is that, in all cases, the satisfaction ratio reaches a high value within the duration of the simulation (95\% with 60 clients). Figures 3 also represents the distribution of the service delivery delays for TAO-INV in the real traces scenario. We notice that the majority of the round trip durations fall in the 1 to 10 seconds period when having 10, 30 and 60 clients in the network. Indeed, since TAO does not rely on an exchange of summary vectors among nodes, it is able to preserve a lower average round trip time than other more general approaches. Concerning the load imposed by TAO over the network, despite the increase in the number of mobile nodes roaming the network, the increase in the number of exchanged messages is limited. The main explanation lies in the fact that sending a request with TAOINVO is adapted to the hybrid characteristics of the network, as it is specialized for sending requests only to the infostations. Indeed, a TAO node only embeds in the beaconing message the last date of contact with the infostation. Moreover, the time heuristic implemented in TAO permits somehow to reflect the notion of distance separating the mobile node from the infostation.

\section{CONClusion}

In this paper, we have presented TAO-INV and TAO-DIS, two protocols dedicated to service provisioning in ICHNs. The originality of these protocols lies in the fact that their design strives to exploit the pattern of communication induced by invocations as well as the fact that an ICHN includes not only intermittently connected mobile clients but also fixed infostations that host service providers. This is achieved mainly thanks to a simple heuristic that exploits the last date of contact between a mobile device and an infostation. Simulation experiments based in particular on real mobility traces data showed that TAO is able to guarantee good performance with limited overhead.

\section{REFERENCES}

[1] M. J. Khabbaz, A. Chadi M., and F. Wissam F., "Disruption-Tolerant Networking: A Comprehensive Survey on Recent Developments and Persisting Challenges," IEEE Communications Surveys and Tutorials, vol. 14, no. 2, pp. 607-640, 2012.

[2] M. Conti and M. Kumar, "Opportunities in Opportunistic Computing," Computer, vol. 43, pp. 42-50, 2010.

[3] W. Zhao, M. Ammar, and E. Zegura, "A Message Ferrying Approach for Data Delivery in Sparse Mobile Ad Hoc Networks," in Proc. of the 5th International Symposium on Mobile Ad Hoc Networking and Computing (Mobihoc 2004). Tokyo, Japan: ACM, May 2004.

[4] A. Vahdat and D. Becker, "Epidemic Routing for Partially-Connected Ad Hoc Networks," Duke University, Tech. Rep., Apr. 2000.

[5] T. Spyropoulos, K. Psounis, and C. S. Raghavendra, "Spray and Wait: an Efficient Routing Scheme for Intermittently Connected Mobile Networks," in Proc. of the ACM SIGCOMM Workshop on Delay-Tolerant Networking (WDTN'05), Philadelphia, PA, USA, 2005, pp. 252-259.

[6] A. Lindgren, A. Doria, and O. Schelén, "Probabilistic Routing in Intermittently Connected Networks," in Proc. of the 1st International Workshop on Service Assurance with Partial and Intermittent Resources (SAPIR'04). Fortaleza, Brazil: Springer, Aug. 2004, pp. 239-254.

[7] C. Boldrini, M. Conti, and A. Passarella, "Context and Resource Awareness in Opportunistic Network Data Dissemination," in Proc. of the 2nd WoWMoM Workshop on Autonomic and Opportunistic Communications (AOC 2008). Newport Beach, CA, USA: IEEE CS, Jun. 2008.

[8] R. Said and Y. Mahéo, "Toward a Platform for Service Discovery and Invocation in Disconnected Mobile Ad Hoc Networks," in Proc. of the 5th International Conference on Embedded and Ubiquitous Computing (EUC 2008). Shanghai, China: IEEE CS, Dec. 2008, pp. 238-244.

[9] Z. Wang, E. Bulut, and B. K. Szymanski, "Service Discovery for Delay Tolerant Networks," in Proc. of the 5th Workshop on Heterogeneous, Multi-Hop, Wireless and Mobile Networks (HeterWMN 2010). Miami, FL, USA: IEEE CS, Dec. 2010, pp. 136-141.

[10] M. Conti, S. Giordano, M. May, and A. Passarella, "From opportunistic networks to opportunistic computing," IEEE Communications Magazine, vol. 48, no. 9, pp. 126-139, Sep. 2010.

[11] N. Le Sommer, S. Ben Sassi, F. Guidec, and Y. Mahéo, "A Middleware Support for Location-Based Service Discovery and Invocation in Disconnected MANETs," Studia Informatica Universalis, vol. 8, no. 3, pp. 71-97, Sep. 2010

[12] Y. Mahéo and R. Said, "Service Invocation over Content-Based Communication in Disconnected Mobile Ad Hoc Networks," in Proc. of the 24th International Conference on Advanced Information Networking and Applications (AINA'10). Perth, Australia: IEEE CS, Apr. 2010, pp. 503-510.

[13] A. Varga, "The OMNeT++ Discrete Event Simulation System," European Simulation Multiconference (ESM'2001), pp. 319-324, 2001.

[14] I. Rhee, M. Shin, S. Hong, K. Lee, S. Kim, and S. Chong, "CRAWDAD data set ncsu/mobilitymodels (v. 2009-07-23)," Downloaded from http://crawdad.cs.dartmouth.edu/ncsu/mobilitymodels, Jul. 2009. 